\title{
Linear-Response Time-Dependent Density-Functional Theory with Pairing Fields
}

Degao Peng, ${ }^{1}$ Helen van Aggelen, ${ }^{1,}{ }^{2}$ Yang Yang, ${ }^{1}$ and Weitao Yang ${ }^{1,3, a)}$

1) Department of Chemistry, Duke University, Durham, North Carolina, United States, 27708

${ }^{2)}$ Ghent University, Department of Inorganic and Physical Chemistry, 9000 Ghent, Belgium

3) Department of Physics, Duke University, Durham, North Carolina, United States, 27708

Recent development in particle-particle random phase approximation (pp-RPA) broadens the perspective on ground state correlation energies (van Aggelen et al. Phys. Rev. A, 88, 030501 (2013)) and $N \pm 2$ excitation energies (Yang, et al. J. Chem. Phys.). So far Hartree-Fock and approximated density-functional orbitals have been utilized to evaluate the pp-RPA equation. In this paper, to further explore the fundamentals and the potential use of pairing matrix dependent functionals, we present the linear-response time-dependent density-functional theory with pairing fields with both adiabatic and frequency-dependent kernels. This theory is related to the density-functional theory and time-dependent density-functional theory for superconductors, but is applied to normal non-superconducting systems for our purpose. Due to the lack of the proof of the one-to-one mapping between the pairing matrix and the pairing field for time-dependent systems, the linear-response theory is established based on the representability assumption of the pairing matrix. The linear response theory justifies the use of approximated density-functionals in the pp-RPA equation. This work sets the fundamentals for future density-functional development to enhance the description of ground state correlation energies and $N \pm 2$ excitation energies.

Keywords: Particle-particle random phase approximation, time-dependent densityfunctional theory, $N \pm 2$ excitation, linear response theory

a) Electronic mail: weitao.yang@duke.edu 


\section{INTRODUCTION}

Particle-particle random phase approximation ${ }^{1-8}$ (pp-RPA) has been a widely-known method in nuclear physics and textbook material ${ }^{9,10}$ to describe pairing vibrations in nuclei. Recent introduction of pp- $\mathrm{RPA}^{11}$ to quantum chemistry demonstrated a very interesting perspective. Pp-RPA is the first density-functional approximation (DFA) to satisfy the flat-plane condition exactly ${ }^{11,12}$, and outperforms traditional direct particle-hole random phase approximation in many aspects ${ }^{13}$. Theoretical analysis reveals that the correlation energy from pp-RPA with Hartree-Fock references is equivalent to ladder-coupled-cluster doubles $^{14,15}$. Additionally, the $N \pm 2$ excitation energies from pp-RPA can be used to capture valence, double, charge transfer, and Rydberg excitations with a potential $O\left(N^{4}\right)$ scaling $^{16}$. Viewed as an approximation to the pairing-matrix-pairing matrix (pp) response function, in this paper we establish the connection of the pp-RPA formalism to the timedependent density-functional theory (TDDFT) for superconductors ${ }^{17,18}$ as a special case for non-superconducting system.

Density-functional theory ${ }^{19-21}$ (DFT) has been a robust ground state electronic structure theory by treating the electronic density,

$$
\rho(\mathbf{r})=\sum_{\sigma}\left\langle\Phi\left|\hat{\psi}^{\dagger}(\mathbf{x}) \hat{\psi}(\mathbf{x})\right| \Phi\right\rangle
$$

instead of the wavefunction, as the basic variable. In Eq. (1), $\mathbf{x}$ is the generalized coordinate that includes both spatial coordinate $\mathbf{r}$ and spin coordinate $\sigma$, while $\hat{\psi}^{\dagger}$ and $\hat{\psi}$ are field creation and annihilation operators in the second quantized form. The time dependent extension of DFT, i.e. TDDFT ${ }^{22-24}$, further enables us to explore the physics and chemistry of excited states. The adiabatic linear-response formalism of $\mathrm{TDDFT}^{23}$ has been a routine method to study particle-hole excitations for various systems with moderate complexity and $\operatorname{accuracy}^{25-30}$

In superconducting systems, due to the non-vanishing pairing matrix,

$$
\kappa\left(\mathbf{x}, \mathbf{x}^{\prime}\right)=\left\langle\Phi\left|\hat{\psi}\left(\mathbf{x}^{\prime}\right) \hat{\psi}(\mathbf{x})\right| \Phi\right\rangle
$$

the density $\rho(\mathbf{r})$ alone does not contain all the properties of the system. Accordingly, the Hohenberg-Kohn theorem ${ }^{19}$ for superconductors in equilibrium at finite temperature was established for singlet ${ }^{31}$ and triplet ${ }^{32}$ pairing interactions, using $\rho(\mathbf{r})$ and $\kappa\left(\mathbf{x}, \mathbf{x}^{\prime}\right)$ as basic 
variables. The corresponding Kohn-Sham model ${ }^{20}$ is also proposed by mapping $\rho(\mathbf{r})$ and $\kappa\left(\mathbf{x}, \mathbf{x}^{\prime}\right)$ to those of a non-interacting system with non-vanishing pairing fields. As for the time-dependent extension, Wacker, Kümmel, and Gross ${ }^{33}$ (WKG) further proved a RungeGross-like theorem ${ }^{22}$ for superconductors, which states that the time evolution of the density $(\rho(\mathbf{r}, t))$, the diagonal component of the singlet pairing matrix $(\kappa(\mathbf{r} \uparrow, \mathbf{r} \downarrow ; t))$, and the current density $(\mathbf{j}(\mathbf{r}, t))$ uniquely determine the scalar potential $(v(\mathbf{r}, t))$, the diagonal component of the singlet pairing field $(D(\mathbf{r} \uparrow, \mathbf{r} \downarrow ; t))$, and the vector potential $(\mathbf{A}(\mathbf{r}, t))$, up to a gauge transformation. Unfortunately, the Runge-Gross-like theorem involving the general pairing matrix of Eq. (2) has not been proved, probably due to the difficulty resulting from the non-locality of $\kappa\left(\mathbf{x}, \mathbf{x}^{\prime}\right)$. In fact, the TDDFT formalism for superconductors of Ref. ${ }^{17}$ and $^{18}$ was built on the adiabatic linear response of singlet pairing matrix of a Kohn-Sham-like system, where the WKG theorem is not applicable. A kernel of the local-density approximation (LDA) which accounts only singlet effects was also proposed as a screened Coulomb potential $^{18,34}$, with numerical results presented in Ref. ${ }^{35}$.

For normal non-superconducting systems such as atoms and molecules, the pairing matrix $\kappa$ is identically zero in the absence of external pairing fields. However, the fluctuation of the pairing matrix, i.e. the pp response function, is non-vanishing, even for a normal system $^{11}$. Such fluctuation is related to $N \pm 2$ excitation energies, where double ionization and double electron attachment processes are involved. The mean-field description of this pairing matrix fluctuation, i.e. $\mathrm{pp}^{\mathrm{RPA}} \mathrm{A}^{9,10}$, has been used to calculate the Auger spectroscopy ${ }^{36-39}$. Recently, Yang et al. ${ }^{16}$ developed a scheme to calculate neutral excitations based on pp-RPA, demonstrating promising results. To capture the effect beyond the mean-field approximation, one can resort to $a b$ initio wavefunction techniques, which utilize correlated ground state wavefunctions and include higher order excitation operators. Double-ionization-potential/double-electron-attachment equation-of-motion coupled-cluster singles and doubles (DIP/DEA-EOM-CC $)^{40,41}$ are examples of such approaches.

However, because of the steep scaling of the $a b$ initio wavefunction method, we are interested in the DFT formalism of the pp response function. In previous works, the kernel in the pp-RPA equation is always the bare Coulomb, where the potential of other approximate functionals with pairing matrix dependence has never been explored. The TDDFT method for superconductors is a closely related theory; however, triplet excitations are totally absent, and the pp response function on normal systems has not been inferred. In this paper, we 
establish the linear-response time-dependent density-functional theory with pairing fields (TDDFT-P) to tackle the $N \pm 2$ excitation problem of non-superconducting systems, with its connection to and extension of the TDDFT for superconductors. Both the adiabatic and non-adibatic versions of linear-response TDDFT-P are explored. Especially, the adiabatic TDDFT-P justified the practice of utilizing orbitals and eigenvalues of common DFAs in

pp-RPA equations ${ }^{11,13,16}$. Such an extension enables us to capture effects beyond mean-field approximations in $N \pm 2$ excitations, and would also make it possible to capture neutral excitations better by suitable approaches ${ }^{16,42}$.

This article is organized as follows. Sec. II reviews the counterparts of the HohenbergKohn theorem and the Kohn-Sham model in superconducting systems. Sec. III establishes the adiabatic linear-response TDDFT-P for systems in the non-superconducting limit. Sec. IV further extends the theory to include frequency-dependent particle-particle kernels. Sec. $\mathrm{V}$ concludes this article.

\section{DFT WITH PAIRING INTERACTIONS}

In this section the theory of time-independent DFT with pairing interactions is reviewed for completeness. The theory is mainly based on the work of Ref. ${ }^{31}$ and $^{32}$. We also extend the definition of the functionals involved.

We consider a general Hamiltonian including a pairing potential (in atomic units),

$$
\hat{H}=\hat{T}+\hat{V}+\hat{D}+\hat{W}
$$

where $\hat{T}$ is the kinetic energy operator,

$$
\hat{T}=-\frac{1}{2} \int d \mathbf{x} \hat{\psi}^{\dagger}(\mathbf{x}) \nabla^{2} \hat{\psi}(\mathbf{x})
$$

$\hat{V}$ is the normal external potential,

$$
\hat{V}=\int d \mathbf{x} v(\mathbf{r}) \hat{\psi}^{\dagger}(\mathbf{x}) \hat{\psi}(\mathbf{x})
$$

$\hat{D}$ is the external pairing field,

$$
\hat{D}=\frac{1}{2} \int d \mathbf{x} d \mathbf{x}^{\prime}\left[D^{*}\left(\mathbf{x}, \mathbf{x}^{\prime}\right) \hat{\psi}\left(\mathbf{x}^{\prime}\right) \hat{\psi}(\mathbf{x})+\text { h.c. }\right]
$$


and $\hat{W}$ is the two electron interaction

$$
\hat{W}=\frac{1}{2} \int d \mathbf{x}_{1} d \mathbf{x}_{1}^{\prime} d \mathbf{x}_{2} d \mathbf{x}_{2}^{\prime} w\left(\mathbf{x}_{1}, \mathbf{x}_{2}, \mathbf{x}_{2}^{\prime}, \mathbf{x}_{1}^{\prime}\right) \hat{\psi}^{\dagger}\left(\mathbf{x}_{1}\right) \hat{\psi}^{\dagger}\left(\mathbf{x}_{2}\right) \hat{\psi}\left(\mathbf{x}_{2}^{\prime}\right) \hat{\psi}\left(\mathbf{x}_{1}^{\prime}\right)
$$

In Eq. (6), h.c. represents the Hermitian conjugate of the previous term. For a superconducting system, $w$ in Eq. (7) includes the phonon - or other medium, such as antiferromagnetic correlation in high temperature superconductors - mediated electron-electron interaction $^{31,32}$, while for a non-superconducting system, $w$ is just the Coulomb potential as the only electron-electron interaction in atoms and molecules in quantum chemistry,

$$
w_{\mathrm{NS}}\left(\mathbf{x}_{1}, \mathbf{x}_{2}, \mathbf{x}_{2}^{\prime}, \mathbf{x}_{1}^{\prime}\right)=\delta\left(\mathbf{x}_{2}, \mathbf{x}_{2}^{\prime}\right) \delta\left(\mathbf{x}_{1}, \mathbf{x}_{1}^{\prime}\right) \frac{1}{\left|\mathbf{r}_{1}-\mathbf{r}_{2}\right|},
$$

where NS stands for non-superconducting. Refer to Ref. ${ }^{31}$ and $^{32}$ for different models of w's in a superconducting system. A physical external pairing field $D$ only exists when the system is juxtaposed to a superconducting material. Here it is just used as a mathematical tool to establish the theory, and we will take the external pairing field at zero limit in the end $^{31,32,43}$. Since $[\hat{D}, \hat{N}] \neq 0$, where $\hat{N}$ is the number operator, the system is not electron number conserving. We will introduce the chemical potential $\mu$ to control the electron number, such that the expectation value of $\hat{H}^{\prime}=\hat{H}-\mu \hat{N}$, i.e. the grand potential $\Omega$, rather than the total energy, is minimized. A generalized ensemble density matrix $\hat{\Gamma}$ could be defined as

$$
\hat{\Gamma}=\sum_{I} \gamma_{I}\left|\Phi_{I}\right\rangle\left\langle\Phi_{I}\right|
$$

where $\gamma_{I}$ 's are non-negative weights that sum to unity, and $\left|\Phi_{I}\right\rangle$ 's are vectors in the Fock space which are linear combinations of vectors in Hilbert spaces associated with different particle numbers. Note that although DFT of superconductors normally treats systems at finite temperature ${ }^{31,32}$, we focus on the zero-temperature formalism as we are interested in its connection to quantum chemistry. Thus $\hat{\Gamma}$ in Eq. (9) is a zero-temperature ensemble rather than a finite-temperature ensemble. Then the density and the pairing matrix of an ensemble $\hat{\Gamma}$ can be expressed as $\rho(\mathbf{r})=\operatorname{Tr}[\hat{\Gamma} \hat{\rho}(\mathbf{r})]$ and

$$
\kappa\left(\mathbf{x}, \mathbf{x}^{\prime}\right)=\operatorname{Tr}\left[\hat{\Gamma} \hat{\psi}\left(\mathbf{x}^{\prime}\right) \hat{\psi}(\mathbf{x})\right]
$$

The zero-temperature ground state grand potential is then the following minimum:

$$
\Omega_{0}\left[v(\mathbf{r})-\mu, D\left(\mathbf{x}, \mathbf{x}^{\prime}\right)\right]=\min _{\hat{\Gamma}} \operatorname{Tr}\left(\hat{\Gamma} \hat{H}^{\prime}\right)
$$


Apart from the normal density $\rho(\mathbf{r})$, the pairing matrix $\kappa\left(\mathbf{x}, \mathbf{x}^{\prime}\right)$ is also important in the presence of an internal pairing interaction from $\hat{W}$ or an external pairing interaction from $\hat{D}$. The pairing matrix has also been called the pairing tensor ${ }^{10}$, the anomalous density ${ }^{17,18}$, or the non-local gap function ${ }^{31}$ in different context. Due to the anticommutation relations of Fermionic field operators, the pairing matrix is always antisymmetric,

$$
\kappa\left(\mathbf{x}, \mathbf{x}^{\prime}\right)=-\kappa\left(\mathbf{x}^{\prime}, \mathbf{x}\right)
$$

As a consequence, only the antisymmetric part of the pairing field $D\left(\mathbf{x}, \mathbf{x}^{\prime}\right)$ will have nonzero contribution to the total energy. We therefore require that the pairing field should also be antisymmetric,

$$
D\left(\mathbf{x}, \mathbf{x}^{\prime}\right)=-D\left(\mathbf{x}^{\prime}, \mathbf{x}\right)
$$

Capelle et $a l .{ }^{32}$ presented the Hohenberg-Kohn theorem of this system, stating that there exists a one-to-one mapping between the ground state densities $\left(\rho(\mathbf{r}), \kappa\left(\mathbf{x}, \mathbf{x}^{\prime}\right)\right)$ and the ensemble operator for the system. So there is a density functional $\Omega_{\mathrm{HK}}\left[\rho(\mathbf{r}), \kappa\left(\mathbf{x}, \mathbf{x}^{\prime}\right)\right]$ that maps the ensemble representable density and pairing matrix to its ground state grand potential. The density and pairing matrix are said to be $v \& D$ ensemble representable if they can come from an ensemble of ground state wavefunction(s) of some external potential $v$ and external pairing potential $D$.

We now further generalize this functional to Fock space $\bar{N}$ representable density and pairing matrices using the Levy constrained search definition ${ }^{44}$,

$$
\begin{aligned}
& \Omega\left[\rho(\mathbf{r}), \kappa\left(\mathbf{x}, \mathbf{x}^{\prime}\right)\right] \\
= & \inf _{\hat{\Gamma} \rightarrow\left(\rho(\mathbf{r}), \kappa\left(\mathbf{x}, \mathbf{x}^{\prime}\right)\right)} \operatorname{Tr}\left(\hat{\Gamma} \hat{H}^{\prime}\right) \\
= & \inf _{\hat{\Gamma} \rightarrow\left(\rho(\mathbf{r}), \kappa\left(\mathbf{x}, \mathbf{x}^{\prime}\right)\right)}\left\{\operatorname{Tr}[\hat{\Gamma}(\hat{T}+\hat{W})]+\int d \mathbf{r}(v(\mathbf{r})-\mu) \rho(\mathbf{r})-\frac{1}{2} \int d \mathbf{x} d \mathbf{x}^{\prime}\left[D^{*}\left(\mathbf{x}, \mathbf{x}^{\prime}\right) \kappa\left(\mathbf{x}^{\prime}, \mathbf{x}\right)+\text { h.c. }\right]\right\}
\end{aligned}
$$

$=F_{\text {Levy }}\left[\rho(\mathbf{r}), \kappa\left(\mathbf{x}, \mathbf{x}^{\prime}\right)\right]+\int d \mathbf{r}(v(\mathbf{r})-\mu) \rho(\mathbf{r})-\frac{1}{2} \int d \mathbf{x} d \mathbf{x}^{\prime}\left[D^{*}\left(\mathbf{x}, \mathbf{x}^{\prime}\right) \kappa\left(\mathbf{x}^{\prime}, \mathbf{x}\right)+\right.$ h.c. $]$.

Note that Fock space $\bar{N}$ representability is different from the fractional- $\bar{N}$ representability in Ref. ${ }^{45}$. The Fock space $\bar{N}$ representable density and pairing matrix in Eqs. (14)-(16) are only required to come from a Fermionic ensemble density matrix (not necessarily of a ground state), a much less constrained condition than the $v \& D$ ensemble representability. The Fock 
space $\bar{N}$ representability and the $v \& D$ representability are the superconducting counterpart of $N$ representability and $v$ representability in conventional $\mathrm{DFT}^{21}$. The universal functional $F_{\text {Levy }}\left[\rho(\mathbf{r}), \kappa\left(\mathbf{x}, \mathbf{x}^{\prime}\right)\right]$ thus contains the kinetic and two-body interaction energy. Alternatively, we can use the Lieb-type definition for this universal functional ${ }^{46}$ :

$$
\begin{aligned}
& F_{\text {Lieb }}\left[\rho(\mathbf{r}), \kappa\left(\mathbf{x}, \mathbf{x}^{\prime}\right)\right] \\
= & \sup _{v(\mathbf{r}), D\left(\mathbf{x}, \mathbf{x}^{\prime}\right)}\left\{\Omega_{0}\left[v(\mathbf{r})-\mu, D\left(\mathbf{x}, \mathbf{x}^{\prime}\right)\right]-\int d \mathbf{r}(v(\mathbf{r})-\mu) \rho(\mathbf{r})+\frac{1}{2} \int d \mathbf{x} d \mathbf{x}^{\prime}\left[D^{*}\left(\mathbf{x}, \mathbf{x}^{\prime}\right) \kappa\left(\mathbf{x}^{\prime}, \mathbf{x}\right)+h . c .\right]\right\},
\end{aligned}
$$

with $\Omega_{0}$ defined in Eq. (11).

Now we assume the non-interacting $v \& D$ ensemble representability of Fock space $\bar{N}$ representable densities and pairing matrices, in which the density and the pairing matrix of an interacting ground state system $(w \neq 0)$ can be represented by a density and a pairing matrix of a non-interacting system $(w=0)$. In other words, for $\rho(\mathbf{r})$ and $\kappa\left(\mathbf{x}, \mathbf{x}^{\prime}\right)$ of an interacting ground state, there is always $\rho_{s}(\mathbf{r})$ and $\kappa_{s}\left(\mathbf{x}, \mathbf{x}^{\prime}\right)$ from a non-interacting system with normal potential $v_{s}(\mathbf{r})$ and and pairing potential $D_{s}\left(\mathbf{x}, \mathbf{x}^{\prime}\right)$ such that

$$
\rho(\mathbf{r})=\rho_{s}(\mathbf{r})
$$

and

$$
\kappa\left(\mathbf{x}, \mathbf{x}^{\prime}\right)=\kappa_{s}\left(\mathbf{x}, \mathbf{x}^{\prime}\right) .
$$

Then we can have the grand potential decomposition, ${ }^{18,31,32,34,43}$

$$
\begin{aligned}
\Omega[\rho, \kappa] & =V[\rho]-\mu N+G[\kappa]+F_{\text {Levy }}[\rho, \kappa] \\
& =T_{s}[\rho, \kappa]+V[\rho]+G[\kappa]+J[\rho]+R[\kappa]+E_{\mathrm{XC}}[\rho, \kappa]-\mu N,
\end{aligned}
$$

where $T_{s}[\rho, \kappa]$ is the kinetic energy of the hypothetical non-interacting system with the same density and the pairing matrix, $V[\rho]=\operatorname{Tr}(\hat{\Gamma} \hat{V}), G[\kappa]=\operatorname{Tr}(\hat{\Gamma} \hat{D}), J[\rho]$ is the mean-field energy of the particle-hole channel (usually called the Hartree term in DFT),

$$
J[\rho]=\frac{1}{2} \int \frac{\rho(\mathbf{r}) \rho\left(\mathbf{r}^{\prime}\right)}{\left|\mathbf{r}-\mathbf{r}^{\prime}\right|} d \mathbf{r} d \mathbf{r}^{\prime},
$$

and $R[\kappa]$ is the mean-field energy of the particle-particle channel

$$
R[\kappa]=\frac{1}{2} \int \frac{\kappa\left(\mathbf{x}, \mathbf{x}^{\prime}\right) \kappa^{*}\left(\mathbf{x}, \mathbf{x}^{\prime}\right)}{\left|\mathbf{r}-\mathbf{r}^{\prime}\right|} d \mathbf{x} d \mathbf{x}^{\prime} .
$$


$E_{\mathrm{XC}}[\rho, \kappa]$ includes all the quantum effects that are absent in the other energy terms,

$$
E_{\mathrm{XC}}[\rho, \kappa]=F_{\mathrm{Levy}}[\rho, \kappa]-T_{s}[\rho, \kappa]-J[\rho]-R[\kappa]=T[\rho, \kappa]+W[\rho, \kappa]-T_{s}[\rho, \kappa]-J[\rho]-R[\kappa]
$$

The corresponding Kohn-Sham-like non-interacting system is governed by the noninteracting Hamiltonian

$$
\hat{H}_{s}=\int d \mathbf{x} \hat{\psi}^{\dagger}(\mathbf{x})\left[-\frac{1}{2} \nabla^{2}+v_{s}(\mathbf{r})\right] \hat{\psi}(\mathbf{x})+\frac{1}{2} \int d \mathbf{x} d \mathbf{x}^{\prime}\left[D_{s}\left(\mathbf{x}, \mathbf{x}^{\prime}\right) \hat{\psi}^{\dagger}(\mathbf{x}) \hat{\psi}^{\dagger}\left(\mathbf{x}^{\prime}\right)+\text { h.c. }\right]
$$

with the non-interacting normal potential

$$
v_{s}(\mathbf{r})=v(\mathbf{r})+\int \frac{\rho\left(\mathbf{r}^{\prime}\right)}{\left|\mathbf{r}-\mathbf{r}^{\prime}\right|} d \mathbf{r}^{\prime}+\left(\frac{\delta E_{\mathrm{XC}}[\rho, \kappa]}{\delta \rho(\mathbf{r})}\right)_{\kappa},
$$

and the non-interacting pairing potential

$$
D_{s}\left(\mathbf{x}, \mathbf{x}^{\prime}\right)=D\left(\mathbf{x}, \mathbf{x}^{\prime}\right)+D_{R}\left(\mathbf{x}, \mathbf{x}^{\prime}\right)+D_{\mathrm{XC}}\left(\mathbf{x}, \mathbf{x}^{\prime}\right)
$$

where

$$
D_{R}\left(\mathbf{x}, \mathbf{x}^{\prime}\right)=\frac{\kappa\left(\mathbf{x}, \mathbf{x}^{\prime}\right)}{\left|\mathbf{r}-\mathbf{r}^{\prime}\right|}
$$

and

$$
D_{\mathrm{XC}}\left(\mathbf{x}, \mathbf{x}^{\prime}\right)=\left(\frac{\delta E_{\mathrm{XC}}[\rho, \kappa]}{\delta \kappa^{*}\left(\mathbf{x}, \mathbf{x}^{\prime}\right)}\right)_{\rho} .
$$

The resulting self-consistent equation for this non-interacting system is the well-known Kohn-Sham Bogoliubov-de Gennes (KS-BdG) equation ${ }^{18,35,47}$, or the Hartree-Fock-Bogoliubov equation for the Hartree-Fock approximate functional ${ }^{10,48}$. The KS-BdG equation and its solution are not necessary to establish the linear response theory in Sec. III and IV, and are thus not addressed here. Refer to standard textbooks such as Ref. ${ }^{9}$ and $^{10}$ for details of the equation and its solution.

\section{ADIABATIC LINEAR-RESPONSE TDDFT-P FOR NON-SUPERCONDUCTING SYSTEMS}

We now establish the theory for adiabatic linear-response TDDFT with pairing fields (TDDFT-P) for non-superconducting systems. This generalizes the previous TDDFT for superconductors with singlet-only excitations ${ }^{17,18}$ to include triplet excitations. The adiabatic linear-response TDDFT-P provides a theoretical foundation for using adiabatic DFAs 
in the pp-RPA equation. Especially, it justifies the application of orbitals and eigenvalues from common DFAs in the pp-RPA equation.

Suppose we perturb the interacting non-superconducting system with a small paring field

$$
\delta D\left(\mathbf{x}, \mathbf{x}^{\prime} ; t\right)=\sum_{p q} \delta D_{p q}(t) \varphi_{p}(\mathbf{x}) \varphi_{q}\left(\mathbf{x}^{\prime}\right)
$$

In this paper we will use $p, q, r, s$ for general orbitals, $i, j, k, l$ for occupied orbitals, and $a$, $b, c, d$ for unoccupied orbitals. For simplicity, we only discuss non-degenerate ground states which can be represented as vectors in the Hilbert space for non-superconducting systems. According to the linear response theor ${ }^{9,11}$, the linear response of the pairing matrix is related to the pairing field by a pp response function,

$$
K\left(\mathbf{x}, \mathbf{x}^{\prime} ; \mathbf{y}, \mathbf{y}^{\prime} ; t\right)=-i \theta(t)\left\langle\Phi^{\mathrm{gs}}\left|\left[\hat{\psi}_{H}\left(\mathbf{x}^{\prime} t\right) \hat{\psi}_{H}(\mathbf{x} t), \hat{\psi}^{\dagger}(\mathbf{y}) \hat{\psi}^{\dagger}\left(\mathbf{y}^{\prime}\right)\right]\right| \Phi^{\mathrm{gs}}\right\rangle
$$

such that

$$
\delta \kappa\left(\mathbf{x}, \mathbf{x}^{\prime} ; t\right)=\int d \tau d \mathbf{y} d \mathbf{y}^{\prime} K\left(\mathbf{x}, \mathbf{x}^{\prime} ; \mathbf{y}, \mathbf{y}^{\prime} ; t-\tau\right) \delta D\left(\mathbf{y}, \mathbf{y}^{\prime} ; \tau\right)
$$

with $\hat{\psi}_{H}(\mathbf{x} t)=e^{i \hat{H}^{\prime} t} \hat{\psi}(\mathbf{x}) e^{-i \hat{H}^{\prime} t}$ the interacting field operator in the Heisenberg picture and $\theta(t)$ the Heaviside step function. Expressed in a one-particle basis and transferred to the frequency domain, Eq. (32) becomes

$$
\delta \kappa_{p q}(\omega)=\sum_{r s} K_{p q, r s}(\omega) \delta D_{r s}(\omega)
$$

with

$$
K_{p q, r s}(\omega)=\int d t e^{i \omega t} d \mathbf{x} d \mathbf{x}^{\prime} d \mathbf{y} d \mathbf{y}^{\prime} K\left(\mathbf{x}, \mathbf{x}^{\prime} ; \mathbf{y}, \mathbf{y}^{\prime} ; t\right) \varphi_{p}^{*}(\mathbf{x}) \varphi_{q}^{*}\left(\mathbf{x}^{\prime}\right) \varphi_{r}(\mathbf{y}) \varphi_{s}\left(\mathbf{y}^{\prime}\right)
$$

The pp response function is related to the correlation energy and $N \pm 2$ excitation energies. ${ }^{11,16}$ Specifically, if we perturb the corresponding KS-BdG system with a small pairing field $\delta D_{s}\left(\mathbf{x}, \mathbf{x}^{\prime} ; t\right)=\sum_{p q} \delta D_{p q}^{s}(t) \varphi_{p}(\mathbf{x}) \varphi_{q}\left(\mathbf{x}^{\prime}\right)$, the pp response function is

$$
K_{0}\left(\mathbf{x}, \mathbf{x}^{\prime} ; \mathbf{y}, \mathbf{y}^{\prime} ; t\right)=-i \theta(t)\left\langle\Phi_{s}^{\mathrm{gs}}\left|\left[\hat{\psi}_{H_{s}}\left(\mathbf{x}^{\prime} t\right) \hat{\psi}_{H_{s}}(\mathbf{x} t), \hat{\psi}^{\dagger}(\mathbf{y}) \hat{\psi}^{\dagger}\left(\mathbf{y}^{\prime}\right)\right]\right| \Phi_{s}^{\mathrm{gs}}\right\rangle,
$$

with $\hat{\psi}_{H_{s}}(\mathbf{x} t)=e^{i \hat{H}_{s}^{\prime} t} \hat{\psi}(\mathbf{x}) e^{-i \hat{H}_{s}^{\prime} t}$ the non-interacting field operator in the interacting picture. The corresponding first order pairing matrix variation is,

$$
\delta \kappa_{s}\left(\mathbf{x}, \mathbf{x}^{\prime} ; t\right)=\int d \tau d \mathbf{y} d \mathbf{y}^{\prime} K_{0}\left(\mathbf{x}, \mathbf{x}^{\prime} ; \mathbf{y}, \mathbf{y}^{\prime} ; t-\tau\right) \delta D_{s}\left(\mathbf{y}, \mathbf{y}^{\prime} ; \tau\right)
$$


For a non-interacting non-superconducting system, $K_{p q, r s}^{0}(\omega)$ is trivial ${ }^{9,11}$,

$$
K_{p q, r s}^{0}(\omega)=\left(\delta_{p r} \delta_{q s}-\delta_{q r} \delta_{p s}\right) \frac{\theta(p-F) \theta(q-F)-\theta(F-p) \theta(F-q)}{\omega-\left(\epsilon_{p}+\epsilon_{q}-2 \mu\right)+i \eta}
$$

where $F$ represents the Fermi level such that $p-F>0$ if $p$ is an unoccupied orbital and $p-F<0$ if $p$ is an occupied orbital. Accordingly, we can express the linear response of the pairing matrix as,

$$
\begin{aligned}
\delta \kappa_{i j}^{s}(\omega) & =-\frac{\delta D_{i j}^{s}(\omega)}{\omega-\left(\epsilon_{i}+\epsilon_{j}-2 \mu\right)+i \eta} \\
\delta \kappa_{a b}^{s}(\omega) & =\frac{\delta D_{a b}^{s}(\omega)}{\omega-\left(\epsilon_{a}+\epsilon_{b}-2 \mu\right)+i \eta},
\end{aligned}
$$

and $\delta \kappa_{i a}^{s}(\omega)=\delta \kappa_{a i}^{s}(\omega)=0 . \eta$ is an infinitesimal positive number to ensure the convergence of the Fourier transformation. For the derivations below, we will drop $\eta$ as it does not affect the resulting equation.

To establish the adiabatic linear-response TDDFT-P, we assume that a) the first order interacting pairing matrix variation $\delta \kappa\left(\mathbf{x}, \mathbf{x}^{\prime} ; \omega\right)$ can be represented by the first order noninteracting pairing matrix variation $\delta \kappa_{s}\left(\mathbf{x}, \mathbf{x}^{\prime} ; \omega\right)$, and that $\left.\mathrm{b}\right)$ the response of $\delta D_{s}\left(\mathbf{x}, \mathbf{x}^{\prime} ; \omega\right)$ to $\delta \kappa\left(\mathbf{y}, \mathbf{y}^{\prime} ; \omega\right)$ is adiabatic. The representability assumption enables us to study a many-body interacting system in terms of its non-interacting KS-BdG system. From now on we will drop the subscript or superscript of $\kappa$ as $\delta \kappa_{s}=\delta \kappa$. Approximation a) is made in analogy to the assumption in conventional TDDFT where the first order density change in the KS system also represents the first order density change in the interacting system, viz $\delta \rho_{s}=\delta \rho$.The conditions under which this assumption is valid are perhaps more restrictive than in DFT, and we consider this an ad hoc assumption that enables us to formulate a linear-response TDDFT-P. Under an external pairing field perturbation of Eq. (30), using Eqs. (37)-(38), we have

$$
\begin{aligned}
-\left[\omega-\left(\epsilon_{i}+\epsilon_{j}-2 \mu\right)\right] \delta \kappa_{i j}(\omega) & =\delta D_{i j}^{s}(\omega) \\
& =\delta D_{i j}(\omega)+\delta D_{i j}^{R}(\omega)+\delta D_{i j}^{\mathrm{XC}}(\omega) \\
& =\delta D_{i j}(\omega)+\sum_{k>l} L_{i j, k l} \delta \kappa_{k l}(\omega)+\sum_{c>d} L_{i j, c d} \delta \kappa_{c d}(\omega),
\end{aligned}
$$

and

$$
\left[\omega-\left(\epsilon_{a}+\epsilon_{b}-2 \mu\right)\right] \delta \kappa_{a b}(\omega)=\delta D_{a b}(\omega)+\sum_{k>l} L_{a b, k l} \delta \kappa_{k l}(\omega)+\sum_{c>d} L_{a b, c d} \delta \kappa_{c d}(\omega)
$$


where the adiabatic pp response kernel is

$$
L_{p q, r s}=\langle p q \| r s\rangle+2 \int d \mathbf{x}_{1} d \mathbf{x}_{2} d \mathbf{x}_{1}^{\prime} d \mathbf{x}_{2}^{\prime} \varphi_{p}^{*}\left(\mathbf{x}_{1}\right) \varphi_{q}^{*}\left(\mathbf{x}_{1}^{\prime}\right)\left(\frac{\delta^{2} E_{\mathrm{XC}}[\rho, \kappa]}{\delta \kappa^{*}\left(\mathbf{x}_{1}, \mathbf{x}_{1}^{\prime}\right) \delta \kappa\left(\mathbf{x}_{2}, \mathbf{x}_{2}^{\prime}\right)}\right)_{\rho} \varphi_{r}\left(\mathbf{x}_{2}\right) \varphi_{s}\left(\mathbf{x}_{2}^{\prime}\right),
$$

with

$$
\langle p q|| r s\rangle=\langle p q \mid r s\rangle-\langle p q \mid s r\rangle
$$

and

$$
\langle p q \mid r s\rangle=\int d \mathbf{x} d \mathbf{x}^{\prime} \frac{\varphi_{p}^{*}(\mathbf{x}) \varphi_{q}^{*}\left(\mathbf{x}^{\prime}\right) \varphi_{r}(\mathbf{x}) \varphi_{s}\left(\mathbf{x}^{\prime}\right)}{\left|\mathbf{r}-\mathbf{r}^{\prime}\right|} .
$$

The adiabatic pp response kernel has the same symmetry as the antisymmetrized twoelectron integral

$$
L_{p q, r s}=-L_{p q, s r}=-L_{q p, r s}=L_{q p, s r}=L_{r s, p q}^{*} .
$$

Then we rearrange Eqs. (39) and (40) using a compact matrix notation,

$$
\left[\begin{array}{cc}
\mathbf{A} & \mathbf{B} \\
\mathbf{B}^{\dagger} & \mathbf{C}
\end{array}\right]\left[\begin{array}{c}
\mathbf{X} \\
\mathbf{Y}
\end{array}\right]-\omega\left[\begin{array}{cc}
\mathbf{I} & \mathbf{0} \\
\mathbf{0} & -\mathbf{I}
\end{array}\right]\left[\begin{array}{c}
\mathbf{X} \\
\mathbf{Y}
\end{array}\right]=-\left[\begin{array}{c}
\delta \mathbf{D}^{p p} \\
\delta \mathbf{D}^{h h}
\end{array}\right]
$$

where

$$
\begin{gathered}
A_{a b, c d}=\left(\epsilon_{a}+\epsilon_{b}-2 \mu\right) \delta_{a c} \delta_{b d}+L_{a b, c d}, \\
B_{a b, i j}=L_{a b, i j}, \\
C_{i j, k l}=-\left(\epsilon_{i}+\epsilon_{j}-2 \mu\right) \delta_{i k} \delta_{j l}+L_{i j, k l}, \\
X_{a b}=\delta \kappa_{a b}(\omega), \\
Y_{i j}=\delta \kappa_{i j}(\omega), \\
{\left[\delta \mathbf{D}^{p p}\right]_{a b}=\delta D_{a b}(\omega),}
\end{gathered}
$$

and

$$
\left[\delta \mathbf{D}^{h h}\right]_{i j}=\delta D_{i j}(\omega)
$$

Note that all matrix indexes require $a>b$ or $i>j$ to eliminate the redundancy and that the two identity matrices I's have different dimensions. With Eq. (45), the pp response function in real-space representation can be expressed as

$$
K\left(\mathbf{x}_{1}, \mathbf{x}_{1}^{\prime} ; \mathbf{x}_{2}, \mathbf{x}_{2}^{\prime} ; \omega\right)=-\sum_{a>b, c>d}\left[\mathbf{M}(\omega)^{-1}\right]_{a b, c d} \Psi_{a b, c d}\left(\mathbf{x}_{1}, \mathbf{x}_{1}^{\prime}, \mathbf{x}_{2}, \mathbf{x}_{2}^{\prime}\right)-\sum_{i>j, k>l}\left[\mathbf{M}(\omega)^{-1}\right]_{i j, k l} \Psi_{i j, k l}\left(\mathbf{x}_{1}, \mathbf{x}_{1}^{\prime}, \mathbf{x}_{2}, \mathbf{x}_{2}^{\prime}\right)
$$


where

$$
\mathbf{M}(\omega)=\left[\begin{array}{cc}
\mathbf{A} & \mathbf{B} \\
\mathbf{B}^{\dagger} & \mathbf{C}
\end{array}\right]-\omega\left[\begin{array}{cc}
\mathbf{I} & \mathbf{0} \\
\mathbf{0} & -\mathbf{I}
\end{array}\right],
$$

and

$$
\begin{aligned}
\Psi_{p q, r s}\left(\mathbf{x}_{1}, \mathbf{x}_{1}^{\prime}, \mathbf{x}_{2}, \mathbf{x}_{2}^{\prime}\right)= & \varphi_{p}\left(\mathbf{x}_{1}\right) \varphi_{q}\left(\mathbf{x}_{1}^{\prime}\right) \varphi_{r}^{*}\left(\mathbf{x}_{2}\right) \varphi_{s}^{*}\left(\mathbf{x}_{2}^{\prime}\right)-\varphi_{p}\left(\mathbf{x}_{1}\right) \varphi_{q}\left(\mathbf{x}_{1}^{\prime}\right) \varphi_{s}^{*}\left(\mathbf{x}_{2}\right) \varphi_{r}^{*}\left(\mathbf{x}_{2}^{\prime}\right) \\
& -\varphi_{q}\left(\mathbf{x}_{1}\right) \varphi_{p}\left(\mathbf{x}_{1}^{\prime}\right) \varphi_{r}^{*}\left(\mathbf{x}_{2}\right) \varphi_{s}^{*}\left(\mathbf{x}_{2}^{\prime}\right)+\varphi_{q}\left(\mathbf{x}_{1}\right) \varphi_{p}\left(\mathbf{x}_{1}^{\prime}\right) \varphi_{s}^{*}\left(\mathbf{x}_{2}\right) \varphi_{r}^{*}\left(\mathbf{x}_{2}^{\prime}\right) .
\end{aligned}
$$

Eq. (45) describes the response of the pairing matrix with respect to the pairing field perturbation, similar to a driven harmonic oscillator. Alternatively, we can study the eigenmode of the system by eliminating the driving force $\delta D$. The resulting eigenvalue equation is

$$
\left[\begin{array}{cc}
\mathbf{A} & \mathbf{B} \\
\mathbf{B}^{\dagger} & \mathbf{C}
\end{array}\right]\left[\begin{array}{l}
\mathbf{X} \\
\mathbf{Y}
\end{array}\right]=\omega\left[\begin{array}{cc}
\mathbf{I} & \mathbf{0} \\
\mathbf{0} & -\mathbf{I}
\end{array}\right]\left[\begin{array}{l}
\mathbf{X} \\
\mathbf{Y}
\end{array}\right],
$$

with the eigenvalues $N+2$ excitation energies

$$
\omega_{n}^{N+2}=\Omega_{n}^{N+2}-\Omega_{0}^{N}=E_{n}^{N+2}-E_{0}^{N}-2 \mu,
$$

and $N-2$ excitation energies

$$
\omega_{n}^{N-2}=\Omega_{0}^{N}-\Omega_{n}^{N-2}=E_{0}^{N}-E_{n}^{N-2}-2 \mu .
$$

These eigenvalues are also the poles of the pp response function in Eq. (53). Eq. (56) can be solved for every interacting strength so the correlation energy can also be covered in the adiabatic-connection fluctuation-dissipation approach, similar to the correlation energy beyond ph-RPA. ${ }^{4-51}$

The accuracy of Eq. (56) relies on how physical the approximation of Eq. (41) is. For a non-superconducting system, both $\kappa$ and $\delta E_{\mathrm{XC}}[\rho, \kappa] / \delta \kappa^{*}$ are zero, thus the only explicit contribution of $E_{\mathrm{XC}}$ to $L$ is

$$
\lim _{\kappa \rightarrow 0} \frac{\delta^{2} E_{\mathrm{XC}}[\rho, \kappa]}{\delta \kappa^{*} \delta \kappa}
$$

The dependency of $E_{\mathrm{XC}}$ with respect to $\kappa$ is non-trivial and not well understood as the exchange-correlation functional in traditional DFT. The simplest approximation to $E_{\mathrm{XC}}[\rho, \kappa]$ is to neglect the pairing matrix dependence,

$$
E_{\mathrm{XC}}^{\mathrm{DFA}}[\rho, \kappa] \equiv E_{\mathrm{XC}}^{\mathrm{DFA}}[\rho, \kappa=0]=E_{\mathrm{XC}}^{\mathrm{DFA}}[\rho]
$$


where $E_{\mathrm{XC}}^{\mathrm{DFA}}[\rho]$ is the common exchange-correlation energy functionals in DFT. When the approximation of Eq. $(60)$ is adopted, $E_{\mathrm{XC}}$ has zero contribution to $L$ and the resulting pp kernel is

$$
L_{p q, r s}^{\mathrm{DFA}}=\langle p q \| r s\rangle
$$

identical to the kernel in pp-RPA ${ }^{9,10}$. The use of Eq. (61) has been a natural consequence of the mean-field approximation in pp-RPA when a Hartree-Fock reference is deployed. Now in view of TDDFT-P, Eq. (61) can be interpreted as a kernel under the approximation of Eq. (60) for any pairing matrix free DFAs. More importantly, the TDDFT-P perspective rationalizes the practice of using orbitals and eigenvalues from common DFAs (such as B3LYP or PBE) in the pp-RPA equation ${ }^{11,13,16}$. If the pp-RPA equation is derived through the equation-of-motion ansatz, since the KS orbitals are not eigenvectors of the Fock matrix, thus the resulting matrix elements will contains the non-diagonal Fock matrix elements in Eqs. (46) and (48). It is clear now that the pp-RPA equation can utilize DFT reference according to the approximation of Eq. (60), which is a main result of this paper.

In the aforementioned approximation, $E_{\mathrm{XC}}$ has zero contribution to $L$. Ref. ${ }^{34}$ and $^{18}$ presented an LDA functional for the pp interaction with non-zero $E_{\mathrm{XC}}$ contribution to $L$, which in principle could be used to calculate $N \pm 2$ excitation energies. However, their LDA is just a Coulomb with screening counting only singlet interactions, which is not the true LDA. The true LDA should include the energies of homogeneous electron gas at different pairing fields, like that in the local-spin-density approximation. Unfortunately, to the best of our knowledge, no quantum Monte Carlo calculations for the homogeneous electron gas were performed under pairing fields. Thus, a true LDA functional accounts for both singlet and triplet interactions is still in need.

In summary, adiabatic linear-response TDDFT-P justifies the use of common pairing matrix independent DFAs in the pp-RPA equation by the approximation of Eq. (60). This approximation leads to the working equation of pp-RPA using common DFAs as practiced in Ref. ${ }^{11,13,16}$. Additionally, TDDFT-P allows other pairing matrix dependent functionals $E_{\mathrm{XC}}[\rho, \kappa]$ to be used to calculate $N \pm 2$ excitations in the pp-RPA equation. 


\section{LINEAR-RESPONSE TDDFT-P WITH FREQUENCY-DEPENDENT PP KERNELS}

Sec. III establishes the theory of adiabatic linear-response TDDFT-P. We demonstrate in this section that the extension beyond the adiabatic approximation can also be formulated by just adopting the same representability approximation as that in Sec. III.

$\mathrm{WKG}^{33}$ proved that there is a one-to-one mapping between the time evolution of $(\rho(\mathbf{r} ; t), \kappa(\mathbf{r} \uparrow, \mathbf{r} \downarrow ; t), \mathbf{j}(\mathbf{r} ; t))$ and the field $(v(\mathbf{r} ; t), D(\mathbf{r} \uparrow, \mathbf{r} \downarrow ; t), \mathbf{A}(\mathbf{r} ; t))$, except for a gauge

transformation. However, the one-to-one mapping involving the general pairing matrix $\kappa\left(\mathbf{x}, \mathbf{x}^{\prime} ; t\right)$ and the general pairing field $D\left(\mathbf{x}, \mathbf{x}^{\prime} ; t\right)$ has not been proved, and the existence of this one-to-one mapping is still unknown. In fact, the linear-response TDDFT for superconductors ${ }^{17,18}$ was built on an approximation of the kernel rather than on the WKG one-to-one mapping.

Due to the absence of the proof of the one-to-one mapping between $\left(\rho(\mathbf{r}, t), \kappa\left(\mathbf{r}, \mathbf{r}^{\prime} ; t\right)\right)$ and $\left(v(\mathbf{r} t), D\left(\mathbf{x}, \mathbf{x}^{\prime} ; t\right)\right)$, it is not straightforward to present the Dyson-like equation of TDDFT-P. If one follows the usual TDDFT derivation, the density-density response function can be expressed $\mathrm{as}^{29}$

$$
\begin{aligned}
\chi\left(\mathbf{r}_{1} t_{1} ; \mathbf{r}_{2} t_{2}\right) & =\frac{\delta \rho\left(\mathbf{r}_{1} t_{1}\right)}{\delta v\left(\mathbf{r}_{2} t_{2}\right)} \\
& =\int d \mathbf{r}_{3} d t_{3} \frac{\delta \rho_{s}\left(\mathbf{r}_{1} t_{1}\right)}{\delta v_{s}\left(\mathbf{r}_{3} t_{3}\right)} \frac{\delta v_{s}\left(\mathbf{r}_{3} t_{3}\right)}{\delta v\left(\mathbf{r}_{2} t_{2}\right)} \\
& =\int d \mathbf{r}_{3} d t_{3} \frac{\delta \rho_{s}\left(\mathbf{r}_{1} t_{1}\right)}{\delta v_{s}\left(\mathbf{r}_{3} t_{3}\right)}\left(\frac{\delta v\left(\mathbf{r}_{3} t_{3}\right)}{\delta v\left(\mathbf{r}_{2} t_{2}\right)}+\frac{\delta\left(v_{s}\left(\mathbf{r}_{3} t_{3}\right)-v\left(\mathbf{r}_{3} t_{3}\right)\right)}{\delta v\left(\mathbf{r}_{2} t_{2}\right)}\right) \\
& =\int d \mathbf{r}_{3} d t_{3} \frac{\delta \rho_{s}\left(\mathbf{r}_{1} t_{1}\right)}{\delta v_{s}\left(\mathbf{r}_{3} t_{3}\right)}\left(\delta\left(\mathbf{r}_{3} t_{3}, \mathbf{r}_{2} t_{2}\right)+\int d \mathbf{r}_{4} d t_{4} \frac{\delta\left(v_{s}\left(\mathbf{r}_{3} t_{3}\right)-v\left(\mathbf{r}_{3} t_{3}\right)\right)}{\delta \rho\left(\mathbf{r}_{4} t_{4}\right)} \frac{\delta \rho\left(\mathbf{r}_{4} t_{4}\right)}{\delta v\left(\mathbf{r}_{2} t_{2}\right)}\right) \\
& =\chi_{s}\left(\mathbf{r}_{1} t_{1} ; \mathbf{r}_{2} t_{2}\right)+\int d \mathbf{r}_{3} d t_{3} d \mathbf{r}_{4} d t_{4} \chi_{s}\left(\mathbf{r}_{1} t_{1} ; \mathbf{r}_{3} t_{3}\right) f_{\mathrm{MB}}\left(\mathbf{r}_{3} t_{3} ; \mathbf{r}_{4} t_{4}\right) \chi\left(\mathbf{r}_{4} t_{4} ; \mathbf{r}_{2} t_{2}\right)
\end{aligned}
$$

where the non-adiabatic kernel representing the many-body (MB) memory effect is

$$
f_{\mathrm{MB}}\left(\mathbf{r}_{3} t_{3} ; \mathbf{r}_{4} t_{4}\right)=\frac{\delta\left(v_{s}\left(\mathbf{r}_{3} t_{3}\right)-v\left(\mathbf{r}_{3} t_{3}\right)\right)}{\delta \rho\left(\mathbf{r}_{4} t_{4}\right)} .
$$

The existence of the derivatives of $v_{s}\left(\mathbf{r}_{3} t_{3}\right)$ and $v\left(\mathbf{r}_{3} t_{3}\right)$ with respect to $\rho\left(\mathbf{r}_{4} t_{4}\right)$ is guaranteed by the Runge-Gross theorem ${ }^{22}$ : there is a one-to-one mapping between $v\left(\mathbf{r}_{3} t_{3}\right)\left(v_{s}\left(\mathbf{r}_{3} t_{3}\right)\right)$ and $\rho\left(\mathbf{r}_{4} t_{4}\right)$ up to an additive merely time-dependent function in the potential. However, in 
deriving the pp response function $\delta \kappa_{s}\left(\mathbf{x}_{1}, \mathbf{x}_{1}^{\prime} ; t_{1}\right) / \delta D\left(\mathbf{x}_{2}, \mathbf{x}_{2}^{\prime} ; t_{2}\right)$ following Eqs. (62)-(66), the difficulty lies in the use of the chain rule in Eq. (65): due to the lack of the proof of the particle-particle counterpart of the Runge-Gross theorem, it is unknown whether the map $D \rightarrow \kappa\left(D_{s} \rightarrow \kappa\right)$ is invertible and thus the corresponding derivative $\delta\left(D_{s}-D\right) / \delta \kappa$ may not be rigorously defined. Therefore, a frequency dependent pp kernel for TDDFT-PF is not as straightforward as that in TDDFT.

We bypass the difficulty of the lack of the proof of the one-to-one mapping by adopting Assumption a) in Sec. III and introducing a special probe of $\delta \tilde{D}$ that is bijectively mapped to $\delta \tilde{\kappa}$. The pp response function so defined is thus invertible and contains the same spectrum of the original pp response function. The final Dyson-like equation is then

$$
\tilde{\mathbf{K}}(\omega)=\tilde{\mathbf{K}}_{s}(\omega)+\tilde{\mathbf{K}}_{s}(\omega) \tilde{\mathbf{L}}(\omega) \tilde{\mathbf{K}}(\omega),
$$

where we have expressed the equation in frequency domain and used matrix multiplication to denote integration, and the frequency dependent pp kernel is

$$
\tilde{\mathbf{L}}(\omega)=\frac{\delta\left(\tilde{\mathbf{D}}_{s}(\omega)-\tilde{\mathbf{D}}(\omega)\right)}{\delta \tilde{\boldsymbol{\kappa}}(\omega)},
$$

and the projected interacting and non-interacting linear-response pp response functions are defined

$$
\tilde{\mathbf{K}}(\omega)=\frac{\delta \tilde{\boldsymbol{\kappa}}(\omega)}{\delta \tilde{\mathbf{D}}(\omega)},
$$

and

$$
\tilde{\mathbf{K}}_{s}(\omega)=\frac{\delta \tilde{\boldsymbol{\kappa}}(\omega)}{\delta \tilde{\mathbf{D}}_{s}(\omega)} .
$$

Refer to Appendix A for mathematical details.

Similar to non-adiabatic linear-response TDDFT where double and higher-rank excitations could be included, with a frequency dependent pp kernel of $\tilde{\mathbf{L}}(\omega)$, the dynamic effects of particle-particle (hole-hole) excitations could be accounted, while in DIP/DAE-EOM-CC methods such effects must come from higher-rank excitations such as 3-particle-1-hole and 4-particle-2-hole excitation operators ${ }^{42,52}$. The Dyson-like equation of Eq. (66) justifies the use of frequency dependent pp kernel in the pp-RPA equation of Eq. (45). Additionally, if we cast the adiabatic approximation in $\tilde{\mathbf{L}}(\omega)$, i.e. using Eq. (41), we recover exactly the same results as in Sec. III. 


\section{CONCLUSIONS}

We establish the linear response time-dependent density-functional theory with pairing fields (TDDFT-P) for non-superconducting systems, for pairing fields of general spins including both singlet and triplet interactions. Although the pairing density is identically zero for a non-superconducting system, its linear response is non-zero and contains important information of $N \pm 2$ excitation energies as well as the correlation energy of the $N$-electron system. Due to the lack of a one-to-one mapping proof of the $\left(\rho(\mathbf{r} t), \kappa\left(\mathbf{x}, \mathbf{x}^{\prime} ; t\right)\right)$ and $\left(v(\mathbf{r} t)-\mu, D\left(\mathbf{x}, \mathbf{x}^{\prime} ; t\right)\right)$, the time dependent response theory is not a straightforward generalization of the normal TDDFT. By assuming that any linear-response pairing matrix generated from a pairing field perturbation of an interacting non-superconducting system can be reproduced by the linear-response pairing matrix generated from some pairing field perturbation of a non-interacting non-superconducting system, the Dyson-like equation for the pp response function is obtained, with a frequency dependent pp kernel. We also present the adiabatic linear-response theory in which the kernel is derived from the second order derivatives of the exchange-correlation energy with respect to the pairing matrix. The adiabatic theory is an extension of the previous TDDFT for superconductors to include triplet excitations ${ }^{18}$, and applies to non-superconducting systems like atoms and molecules. TDDFT-P can be a useful theory to capture $N \pm 2$ excitations and correlation energies ${ }^{16}$, going beyond the simplest pp-RPA.

Furthermore, TDDFT-P rationalizes the use of orbitals and eigenvalues directly from DFAs in the pp-RPA equation. The singlet-only LDA functional for superconductors in Ref. $^{34}$ and ${ }^{18}$ could be used for TDDFT-PF calculations, but this functional does not include triplet state information and will probably not be that useful for this purpose. With better approximation of the pp kernel, one can have more accurate $N \pm 2$ excitation energies and ground state correlation energies, and even important neutral excitation energies.

\section{ACKNOWLEDGMENTS}

Support from the Office of Naval Research (ONR) (N00014-09-1-0576), and the National Science Foundation (NSF) (CHE-09-11119) is gratefully appreciated. D.P. has also been supported by the William Krigbaum and Marcus Hobbs Fellowship from Duke University. 
H.v.A. appreciates the support from the FWO-Flanders (Scientific Research Fund Flanders). Y.Y has been supported by the Paul M. Gross Fellowship from Duke University.

\section{REFERENCES}

${ }^{1}$ N. Fukuda, F. Iwamoto, and K. Sawada, Phys. Rev. 135, A932 (1964).

${ }^{2}$ W. J. Mulhall, R. J. Liotta, J. A. Evans, and R. P. Perazzo, Nucl. Phys. A 93, 261 (1967).

${ }^{3}$ D. J. Rowe, Phys. Rev. 175, 1283 (1968).

${ }^{4}$ G. Ripka and R. Padjen, Nucl. Phys. A 132, 489 (1969).

${ }^{5}$ D. J. Rowe, Rev. Mod. Phys. 40, 153 (1968).

${ }^{6}$ J. Vary and J. N. Ginocchio, Nucl. Phys. A 166, 479 (1971).

${ }^{7}$ J. C. Pacheco and N. Vinh Mau, Phys. Rev. C 65, 044004 (2002).

${ }^{8}$ G. Blanchon, N. V. Mau, A. Bonaccorso, M. Dupuis, and N. Pillet, Phys. Rev. C 82, $034313(2010)$.

${ }^{9} \mathrm{~J}$. Blaizot and G. Ripka, Quantum theory of finite systems (Cambridge, MA, 1986).

${ }^{10}$ P. Ring and P. Schuck, The Nuclear Many-Body Problem (Springer, 2004).

${ }^{11}$ H. van Aggelen, Y. Yang, and W. Yang, Phys. Rev. A 88, 030501 (2013).

${ }^{12}$ P. Mori-Sánchez, A. Cohen, and W. Yang, Phys. Rev. Lett. 102, 066403 (2009).

${ }^{13}$ Y. Yang, H. van Aggelen, S. N. Steinmann, D. Peng, and W. Yang, J. Chem. Phys. 139, 174110 (2013).

${ }^{14}$ D. Peng, S. N. Steinmann, H. van Aggelen, and W. Yang, J. Chem. Phys. 139, 104112 (2013).

${ }^{15}$ G. E. Scuseria, T. M. Henderson, and I. W. Bulik, J. Chem. Phys. 139, 104113 (2013).

${ }^{16}$ Y. Yang, H. van Aggelen, and W. Yang, J. Chem. Phys. 139, 224105 (2013).

${ }^{17}$ M. Lüders and E. Gross, Int. J. Quantum Chem. 56, 521 (1995).

${ }^{18}$ M. Lüders, M. Marques, N. Lathiotakis, A. Floris, G. Profeta, L. Fast, A. Continenza,

S. Massidda, and E. K. U. Gross, Phys. Rev. B 72, 24545 (2005).

${ }^{19}$ P. Hohenberg and W. Kohn, Phys. Rev. 136, B864 (1964).

${ }^{20}$ W. Kohn and L. Sham, Phys. Rev. 385, A1133 (1965).

${ }^{21}$ R. G. Parr and W. Yang, Density-Functional Theory of Atoms And Molecules (Oxford University Press, New York, 1989).

${ }^{22}$ E. Runge and E. K. U. Gross, Phys. Rev. Lett. 52, 997 (1984). 
${ }^{23}$ M. E. Casida, in Recent advances in computational chemistry, Vol. 1, edited by D. P. Chong (World Scientific, Singapore, 1995) p. 155.

${ }^{24}$ R. van Leeuwen, Phys. Rev. Lett. 82, 3863 (1999).

${ }^{25}$ S. Hirata and M. Head-Gordon, Chem. Phys. Lett. 302, 375 (1999).

${ }^{26}$ M. a. L. Marques and E. K. U. Gross, Annu. Rev. Phys. Chem. 55, 427 (2004).

${ }^{27}$ M. Marques, Time-Dependent Density Functional Theory, Lecture Notes in Physics (Springer, 2006).

${ }^{28}$ M. E. Casida, J. Mol. Struc.-THEOCHEM 914, 3 (2009).

${ }^{29}$ M. A. L. Marques, N. T. Maitra, and F. M. S. Nogueira, Fundamentals of Time-Dependent Density Functional Theory, Lecture Notes in Physics (Springer Berlin Heidelberg, 2012).

${ }^{30}$ C. A. Ullrich, Time-Dependent Density-Functional Theory: Concepts and Applications, Oxford Graduate Texts (OUP Oxford, 2012).

${ }^{31}$ L. N. Oliveira, E. K. U. Gross, and W. Kohn, Phys. Rev. Lett. 60, 2430 (1988).

${ }^{32}$ K. Capelle and E. K. U. Gross, Int. J. Quantum Chem. 61, 325 (1997).

${ }^{33}$ O. J. Wacker, R. Kümmel, and E. K. U. Gross, Phys. Rev. Lett. 73, 2915 (1994).

${ }^{34}$ S. Kurth, M. Marques, M. Lüders, and E. K. U. Gross, Phys. Rev. Lett. 83, 2628 (1999).

${ }^{35}$ M. Lüders and E. K. U. Gross, Density Functional Theory for Superconductors: A first principles approach to the superconducting phase, Ph.D. thesis (1998).

${ }^{36}$ C.-M. Liegener, Chem. Phys. Lett. 90, 188 (1982).

${ }^{37}$ C.-M. Liegener, J. Chem. Phys. 79, 2924 (1983).

${ }^{38}$ C.-M. Liegener, J. Chem. Phys. 104, 2940 (1996).

${ }^{39}$ J. V. Ortiz, J. Chem. Phys. 81, 5873 (1984).

${ }^{40}$ M. Nooijen and R. J. Bartlett, J. Chem. Phys. 106, 6441 (1997).

${ }^{41}$ M. Nooijen, Int. J. Mol. Sci. 3, 656 (2002).

${ }^{42}$ J. Shen and P. Piecuch, J. Chem. Phys. 138, 194102 (2013).

${ }^{43}$ E. K. U. Gross and S. Kurth, Int. J. Quantum Chem. 40, 289 (1991).

${ }^{44}$ M. Levy, Proc. Natl. Acad. Sci. U. S. A. 76, 6062 (1979).

${ }^{45}$ B. Verstichel, H. van Aggelen, D. Van Neck, P. W. Ayers, and P. Bultinck, J. Chem. Phys. 132, 114113 (2010).

${ }^{46}$ E. H. Lieb, Int. J. Quantum Chem. 24, 243 (1983).

${ }^{47}$ M. Marques and E. K. U. Gross, Density Functional Theory for Superconductors: Exchange and Correlation Potentials for Inhomogeneous Systems, Ph.D. thesis (2000). 
${ }^{48}$ J. G. Valatin, Phys. Rev. 122, 1012 (1961).

${ }^{49}$ F. Furche and T. Van Voorhis, The Journal of chemical physics 122, 164106 (2005).

${ }^{50}$ A. Heß elmann and A. Görling, Molecular Physics 109, 2473 (2011).

${ }^{51}$ T. Gould and J. F. Dobson, J. Chem. Phys. 138, 014109 (2013).

${ }^{52}$ K. W. Sattelmeyer, H. F. Schaefer III, and J. F. Stanton, Chem. Phys. Lett. 378, 42 (2003).

${ }^{53}$ S. Roman, Advanced Linear Algebra, Graduate texts in mathematics (Springer Science+Business Media, LLC, 2008).

${ }^{54}$ A. Ben-Israel and T. N. E. Greville, Generalized Inverses: Theory and Applications (Springer, 2003).

${ }^{55}$ W. Yang, P. Ayers, and Q. Wu, Phys. Rev. Lett. 92, 146404 (2004).

\section{Appendix A: Mathematical Details of Non-Adiabatic Linear-Response TDDFT-P}

In absence of the proof of the time-dependent one-to-one mapping between the pairing matrix and the pairing field, the the inclusion of the frequency dependent effects can merely be introduced based on some assumption. We adopt Assumption a) in Sec. III; i.e., we assume that at the zero pairing field limit, any first order pairing matrix of an interacting non-superconducting system induced by a pairing field can be reproduced by the first order pairing matrix induced by a pairing field in a non-interacting non-superconducting system. Concisely, it can be expressed as following: for every $\delta \mathbf{D}(\omega)$, there exists a $\delta \mathbf{D}_{s}(\omega)$ such that

$$
\delta \boldsymbol{\kappa}(\omega)=\mathbf{K}(\omega) \delta \mathbf{D}(\omega)=\mathbf{K}_{s}(\omega) \delta \mathbf{D}_{s}(\omega),
$$

where we have used matrix multiplication to represent integrals. In this way, $\delta \mathbf{D}_{s}(\omega), \delta \mathbf{D}(\omega)$, and $\delta \boldsymbol{\kappa}(\omega)$ are vectors in the linear space $\mathcal{V}$ of general functions $g\left(\mathbf{x}, \mathbf{x}^{\prime}\right)$ of two points. Then $\mathbf{K}_{s}(\omega)$ and $\mathbf{K}(\omega)$ are linear operators on $\mathcal{V}$. In the language of linear algebra (see, for example, Ref. ${ }^{53}$ ), the aforementioned assumption is equivalent to

$$
\operatorname{Im} \mathbf{K}(\omega) \subseteq \operatorname{Im}_{s}(\omega)
$$

where $\operatorname{Im} \mathbf{S}$ is the image of a linear operator $\mathbf{S}$,

$$
\operatorname{Im} \mathbf{S}=\{\mathbf{v} \in \mathcal{V} \mid \mathbf{v}=\mathbf{S u}, \mathbf{u} \in \mathcal{V}\}
$$


This linear-response representability is less restrictive than the full representability, as the full representability of $\boldsymbol{\kappa}(\omega)$ requires response of all orders should equal. This is the only assumption necessary to establish the Dyson-like equation for non-adiabatic linear-response TDDFT-P. Note that the particle-hole blocks in $\mathbf{K}_{s}(\omega)$ and $\delta \boldsymbol{\kappa}(\omega)$ are zero, as indicated in Eq. (36). Therefore $\mathbf{K}_{s}(\omega)$ is rank deficient and not invertible, and so is $\mathbf{K}(\omega)$. The non-invertibility makes it difficult to derive the Dyson-like equation in the straightforward way as stated in Sec. IV, yet we can bypass such difficulty by restricting the perturbing field. Also note that although there is no particle-hole terms in $\delta \boldsymbol{\kappa}(\omega)$ due to the rank deficiency of $\mathbf{K}_{s}(\omega)$ in Eq. (A1), the full response $\boldsymbol{\kappa}(\omega)$ could have a non-zero particle-hole block.

We perturb the interacting non-superconducting system with a specially designed pairing field $\delta \tilde{\mathbf{D}}(\omega)$ such that

$$
\delta \tilde{\mathbf{D}}(\omega) \in \operatorname{Im} \mathbf{K}^{-}(\omega),
$$

where $\mathbf{K}^{-}(\omega)$ is the Moore-Penrose pseudoinverse (see, for example, Ref. ${ }^{54}$ ) of matrix $\mathbf{K}(\omega)$. Such pairing perturbation will generate first order pairing matrix

$$
\delta \tilde{\boldsymbol{\kappa}}(\omega)=\mathbf{K}(\omega) \delta \tilde{\mathbf{D}}(\omega)
$$

According to Eq. (A1), there are infinitely many $\delta \mathbf{D}_{s}(\omega)$ 's to satisfy the conditions due to the rank deficiency of $\mathbf{K}_{s}(\omega)$ described above. However, we can choose a specific solution $\delta \tilde{\mathbf{D}}_{s}(\omega)$ that fulfills Eq. (A1),

$$
\delta \tilde{\mathbf{D}}_{s}(\omega)=\mathbf{K}_{s}^{-}(\omega) \delta \tilde{\boldsymbol{\kappa}}(\omega)=\mathbf{K}_{s}^{-}(\omega) \mathbf{K}(\omega) \delta \tilde{\mathbf{D}}(\omega)
$$

without ambiguity. Owing to the constraint of Eq. (A4), the pairing field can also be expressed by the first order pairing matrix perturbation

$$
\delta \tilde{\mathbf{D}}(\omega)=\mathbf{K}^{-}(\omega) \delta \tilde{\boldsymbol{\kappa}}(\omega)
$$

Now we introduce a projected linear-response functions

$$
\tilde{\mathbf{K}}(\omega)=\frac{\delta \tilde{\boldsymbol{\kappa}}(\omega)}{\delta \tilde{\mathbf{D}}(\omega)},
$$

and

$$
\tilde{\mathbf{K}}_{s}(\omega)=\frac{\delta \tilde{\boldsymbol{\kappa}}(\omega)}{\delta \tilde{\mathbf{D}}_{s}(\omega)},
$$

which only response to the selected fields $\delta \tilde{\mathbf{D}}(\omega)$ and $\delta \tilde{\mathbf{D}}_{s}(\omega)$. When acting on $\delta \tilde{\mathbf{D}}(\omega)$ $\left(\delta \tilde{\mathbf{D}}_{s}(\omega)\right), \tilde{\mathbf{K}}\left(\tilde{\mathbf{K}}_{s}\right)$ gives the same result as $\mathbf{K}(\omega)\left(\mathbf{K}_{s}(\omega)\right)$. 
Defining $\delta \tilde{\mathbf{D}}_{\mathrm{MB}}(\omega)=\delta \tilde{\mathbf{D}}_{s}(\omega)-\delta \tilde{\mathbf{D}}(\omega)$, where the subscript MB denotes many-body effects, we have

$$
\delta \tilde{\mathbf{D}}_{\mathrm{MB}}(\omega)=\left[\tilde{\mathbf{K}}_{s}^{-}(\omega)-\tilde{\mathbf{K}}^{-}(\omega)\right] \delta \tilde{\boldsymbol{\kappa}}(\omega),
$$

which enables us to define the functional derivative

$$
\tilde{\mathbf{L}}(\omega)=\frac{\delta\left(\tilde{\mathbf{D}}_{s}(\omega)-\tilde{\mathbf{D}}(\omega)\right)}{\delta \tilde{\boldsymbol{\kappa}}(\omega)}=\frac{\delta \tilde{\mathbf{D}}_{\mathrm{MB}}(\omega)}{\delta \tilde{\boldsymbol{\kappa}}(\omega)}=\tilde{\mathbf{K}}_{s}^{-}(\omega)-\tilde{\mathbf{K}}^{-}(\omega)
$$

as the general frequency-dependent pp response kernel. Then, we can express the Dyson-like equation

$\tilde{\mathbf{K}}(\omega)=\frac{\delta \tilde{\boldsymbol{\kappa}}(\omega)}{\delta \tilde{\mathbf{D}}(\omega)}=\frac{\delta \tilde{\boldsymbol{\kappa}}(\omega)}{\delta \tilde{\mathbf{D}}_{s}(\omega)} \frac{\delta \tilde{\mathbf{D}}_{s}(\omega)}{\delta \tilde{\mathbf{D}}(\omega)}=\tilde{\mathbf{K}}_{s}\left(\mathbf{I}+\frac{\delta \tilde{\mathbf{D}}_{\mathrm{MB}}(\omega)}{\delta \tilde{\boldsymbol{\kappa}}(\omega)} \frac{\delta \tilde{\boldsymbol{\kappa}}(\omega)}{\delta \tilde{\mathbf{D}}(\omega)}\right)=\tilde{\mathbf{K}}_{s}(\omega)+\tilde{\mathbf{K}}_{s}(\omega) \tilde{\mathbf{L}}(\omega) \tilde{\mathbf{K}}(\omega)$.

The multiplication by $\delta \tilde{D}_{s}$ and its inverse in the second step are allowed because from Eqs. (A2), (A6) and (A7) it follows that all quantities are in $\operatorname{ImK}^{-}(\omega)$. With only the assumption that the first order pairing matrix of an interacting system can be represented by a first order pairing matrix of some non-interacting system, we obtain the Dyson-like equation with a frequency-dependent pp kernel, which is not necessarily a second order derivative of some functional. Note that if the original $\delta \mathbf{D}_{s}(\omega)$ and $\delta \boldsymbol{\kappa}(\omega)$ were used, the derivative of Eq. (A11) would be undefined since $\delta \mathbf{D}_{s}(\omega)$ and $\delta \mathbf{D}(\omega)$ are not necessarily a function of $\delta \boldsymbol{\kappa}(\omega)$.

It is essential to introduce $\delta \tilde{\mathbf{D}}(\omega)$ in the way expressed in Eq. (A4). We bypass the difficulty of the absence of a one-to-one mapping in Eq. (A1) by choosing a specific type of perturbation in Eq. (A4). Although there is no one-to-one mapping between $\delta \mathbf{D}(\omega)$ and $\delta \boldsymbol{\kappa}(\omega)$, there is a one-to-one mapping between $\delta \tilde{\mathbf{D}}(\omega)$ and $\delta \tilde{\boldsymbol{\kappa}}(\omega)$ as shown in Eqs. (A5) and (A7). For every $\delta \tilde{\mathbf{D}}(\omega)$ that satisfies Eq. (A5), any $\delta \tilde{\mathbf{D}}^{\prime}=\mathbf{s}+\delta \tilde{\mathbf{D}}(\omega)$ with $\mathbf{s} \in \operatorname{KerK}(\omega)$ is also a solution of Eq. (A5), where $\operatorname{KerK}(\omega)$ is the kernel (or the null space) ${ }^{53}$ of the linear operator $\mathbf{K}(\omega)$,

$$
\operatorname{Ker} \mathbf{K}(\omega)=\{\mathbf{x} \in \mathcal{V} \mid \mathbf{K}(\omega) \mathbf{x}=0\}
$$

The constraint of Eq. (A4) specifies the solution associated with s $=0$ so that the one-toone mapping between $\delta \tilde{\mathbf{D}}(\omega)$ and $\delta \tilde{\boldsymbol{\kappa}}(\omega)$ can be established. Using the Hohenberg-Kohn ${ }^{19}$ or Runge-Gross ${ }^{22}$ language, we can say that $\delta \mathbf{D}(\omega)$ can be uniquely determined from $\delta \boldsymbol{\kappa}(\omega)$, up to an unimportant additive vector $\mathbf{s} \in \operatorname{Ker} \mathbf{K}(\omega)$. Figure 1a illustrates the relationship of this mapping. Each distinct vector $\mathbf{s} \in \operatorname{Ker} \mathbf{K}(\omega)$ generates a unique coset (represented 
as an oval in Figure 1a) of $\mathbf{s}+\operatorname{Im} \mathbf{K}^{-}(\omega)=\left\{\mathbf{s}+\mathbf{x} \mid \mathbf{x} \in \operatorname{Im} \mathbf{K}^{-}(\omega)\right\}$, the image of which is exactly $\operatorname{Im} \mathbf{K}(\omega)$ because

$$
\begin{aligned}
& \left\{\mathbf{y} \in \mathcal{V} \mid \mathbf{y}=\mathbf{K}(\omega)(\mathbf{s}+\mathbf{x}), \mathbf{x} \in \operatorname{Im}^{-}(\omega), \mathbf{s} \in \operatorname{Ker} \mathbf{K}(\omega)\right\} \\
= & \left\{\mathbf{y} \in \mathcal{V} \mid \mathbf{y}=\mathbf{K}(\omega) \mathbf{x}, \mathbf{x} \in \operatorname{Im} \mathbf{K}^{-}(\omega)\right\}=\operatorname{Im} \mathbf{K}(\omega) .
\end{aligned}
$$

We design the perturbation $\delta \tilde{\mathbf{D}}(\omega)$ such that it belongs to the coset associated with $\mathbf{s}=\mathbf{0}$, hence a one-to-one mapping (isomorphism) between $\delta \tilde{\mathbf{D}}(\omega)$ and $\delta \tilde{\boldsymbol{\kappa}}(\omega)$ can be established. Figure $1 \mathrm{~b}$ shows the density-density response function map, i.e. the linear order of the Runge-Gross map ${ }^{22}$, as an analogy to the $\mathbf{K}(\omega)$ map in this paper. For the linear order of the Runge-Gross map, i.e.

$$
\delta \boldsymbol{\rho}(\omega)=\chi(\omega) \delta \mathbf{v}(\omega)
$$

there is no one-to-one mapping between $\delta \boldsymbol{\rho}(\omega)$ and $\delta \mathbf{v}(\omega)$, since $\mathbf{c}(\omega)+\delta \mathbf{v}(\omega)$ with $\mathbf{c}(\omega)$ any spatial independent vector will not generate a new $\delta \boldsymbol{\rho}(\omega)$ which is generated by $\delta \mathbf{v}(\omega)$. Note that $\delta \boldsymbol{\rho}(\omega)$ and $\delta \mathbf{v}(\omega)$ now represent functions with one coordinate index $\delta \rho(\mathbf{r}, \omega)$ and $\delta v(\mathbf{r}, \omega)$ as they are both local, different from the non-local $\delta \boldsymbol{\kappa}(\omega)$ and $\delta \mathbf{D}(\omega)$ with two coordinate indexes. Nevertheless, we can achieve the one-to-one mapping between $\delta \tilde{\mathbf{v}}(\omega)$ and $\delta \tilde{\boldsymbol{\rho}}(\omega)$ by designing the perturbation $\delta \tilde{\mathbf{v}}(\omega)$ such that $\delta \tilde{\mathbf{v}}(\omega) \in \operatorname{Im} \chi^{-}(\omega)$. Such a potential-centric perspective is an example of the potential functional in Ref. ${ }^{55}$. 


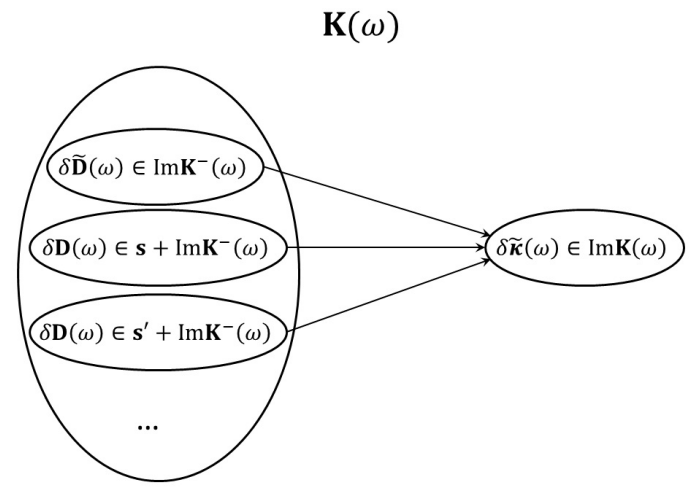

(a) The $\mathbf{K}(\omega)$ map

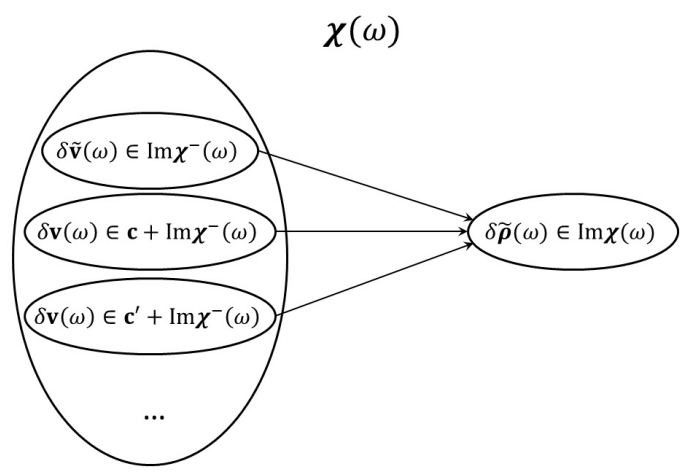

(b) The $\chi(\omega)$ map

Figure 1: The illustration of the one-to-one mapping between (a) $\delta \tilde{\mathbf{D}}(\omega)$ and $\delta \tilde{\boldsymbol{\kappa}}(\omega)$, and (b) $\delta \tilde{\mathbf{v}}(\omega)$ and $\delta \tilde{\boldsymbol{\rho}}(\omega)$. In (a), s and $\mathbf{s}^{\prime}$ are different vectors in $\operatorname{Ker} \mathbf{K}(\omega)$. The small ovals in the domain represents different cosets. A coset is defined as $\mathbf{s}+\operatorname{ImK}^{-}(\omega)=\{\mathbf{s}+\mathbf{x} \mid \mathbf{x} \in$ $\left.\operatorname{Im} \mathbf{K}^{-}(\omega)\right\}$. The image of each coset is the full image $\operatorname{Im} \mathbf{K}(\omega)$. We design the perturbation such that $\delta \tilde{\mathbf{D}}(\omega)$ only resides in one single oval, thus there is a one-to-one mapping between $\delta \tilde{\mathbf{D}}(\omega)$ and $\delta \tilde{\boldsymbol{\kappa}}(\omega)$, despite the overall many-to-one mapping between $\delta \mathbf{D}(\omega)$ and $\delta \tilde{\boldsymbol{\kappa}}(\omega)$. An analogy is present in (b) for the $\boldsymbol{\chi}(\omega)$ map. In this case, $\mathbf{c}$ and $\mathbf{c}^{\prime}$ are different vectors in $\operatorname{Ker} \chi(\omega)$. Any spatial coordinate independent vector $\mathbf{c}$ belongs to $\operatorname{Ker} \chi(\omega)$ and generates a coset $\mathbf{c}+\operatorname{Im} \chi^{-}(\omega)$. The one-to-one mapping between $\delta \tilde{\mathbf{v}}(\omega)$ and $\delta \tilde{\boldsymbol{\rho}}(\omega)$ is fulfilled by designing the perturbation $\delta \tilde{\mathbf{v}}(\omega)$. 


\section{$\mathbf{K}(\omega)$}

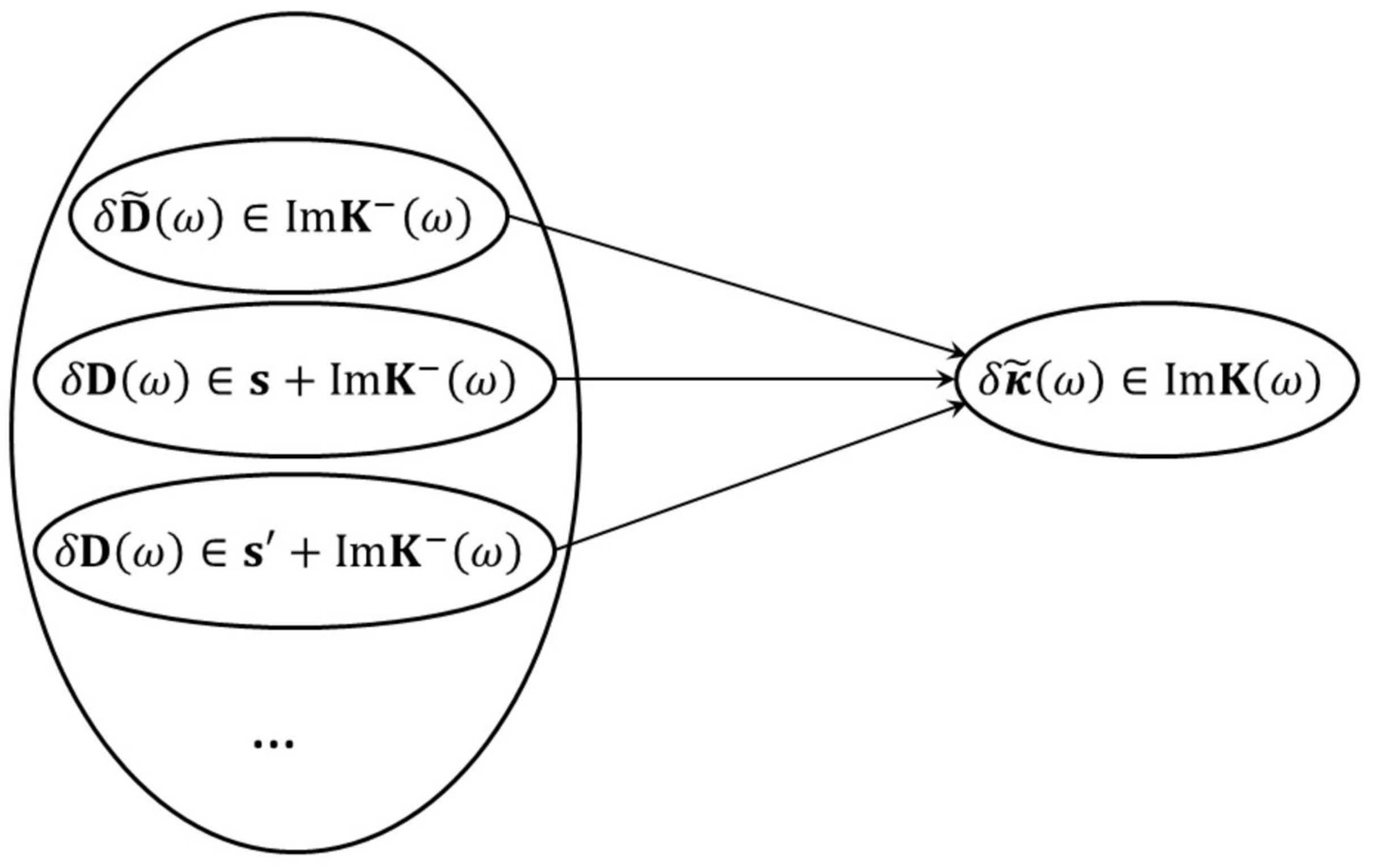




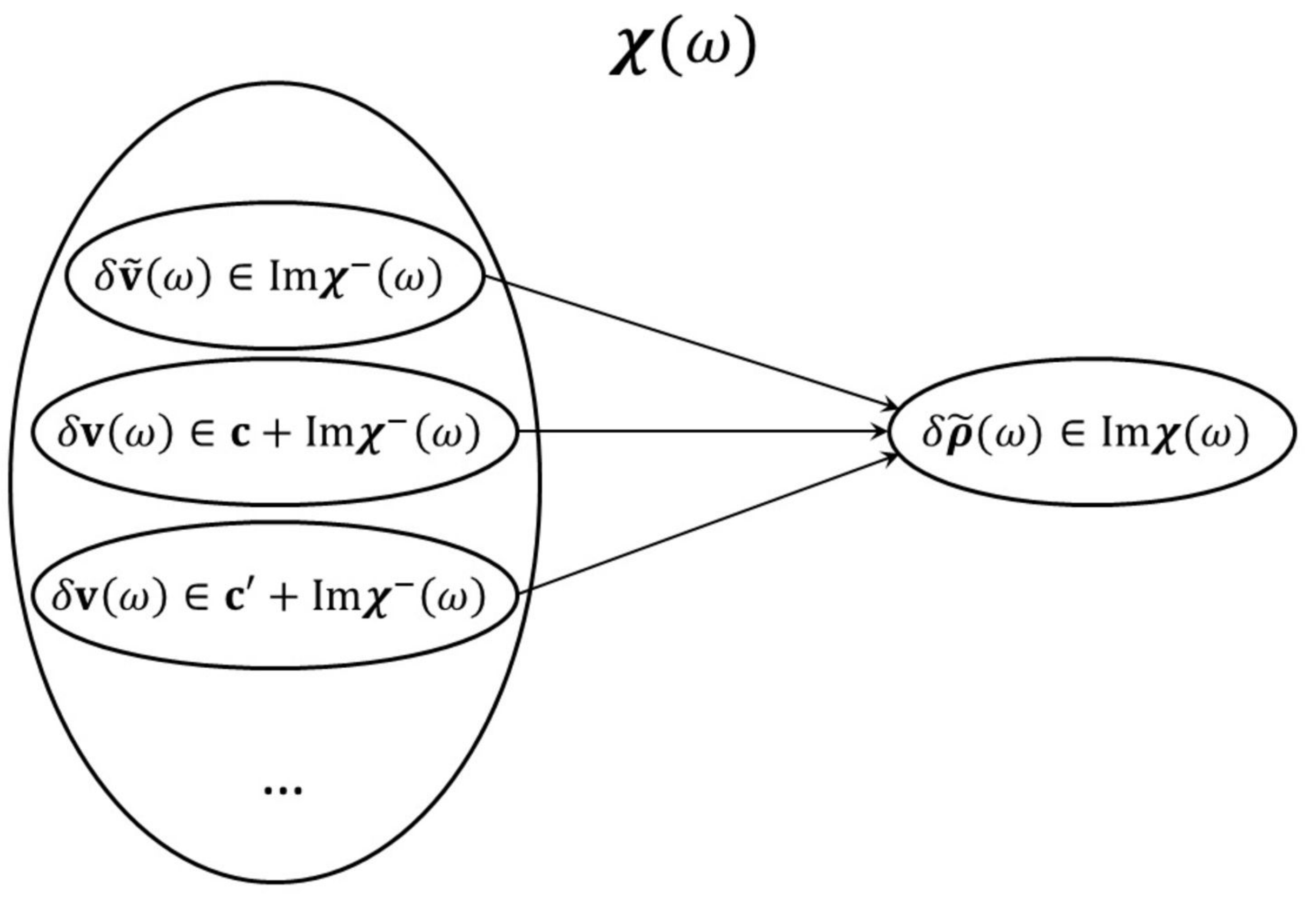

